\title{
Alterações no comportamento da marcha após o uso da estimulação elétrica neuromuscular em pacientes com seqüela de paralisia cerebral do tipo hemiparesia espástica \\ Gait alterations after the use of neuromuscular electrical stimulation in patients with sequels of spastic hemiparetic cerebral palsy
}

Paula Pereira Ferrari, Ft.*, Adriano Rodrigues de Oliveira, Ft.*, Fernanda Ishida Corrêa, M.Sc.**, Renata Calhes Franco, M.Sc.**, João Carlos Ferrari Corrêa, D.Sc.***

*UNINOVE São Paulo SP, **Professora da UNINOVE, Departamento de Ciências da Saúde -Curso de Fisioterapia, São PauloSP, ***Professor do Mestrado em Ciências da Reabilitação da UNINOVE São Paulo SP

\section{Resumo}

Uma das alteraçóes mais importantes da marcha de pacientes com seqüela de paralisia cerebral do tipo hemiparesia espástica é o pé eqüino, que, em geral, é causado por fraqueza dos músculos dorsiflexores. Diversas abordagens não invasivas vem sendo utilizadas no tratamento destas crianças, contudo a estimulação elétrica neuromuscular (EENM) não tem sido freqüentemente reportada como um recurso utilizado na terapia destes pacientes, a qual teria por objetivo o recondicionamento muscular, a redução de espasticidade e o auxílio na aprendizagem motora. Este trabalho foi idealizado, portanto, no intuito de verificar as possíveis alterações no comportamento das forças reaçáo do solo durante a marcha de crianças portadoras de paralisia cerebral do tipo hemiparética espástica, imediatamente após o uso de EENM aplicadas sobre o músculo tibial anterior. Neste estudo foram selecionadas 6 crianças, com idade média de 7,83 \pm 3,60 anos, em que foram aplicadas a freqüência de $50 \mathrm{~Hz}$ no músculo tibial anterior com avaliação do padrão de marcha na plataforma de pressão pré e imediatamente pós EENM. Através dos resultados observou-se que uma única aplicação de EENM não demonstra melhora do comportamento da marcha, quando comparado pré e imediatamente pós EENM, entretanto, fazse necessário a continuaçáo desse estudo com ênfase em aprendizado motor, intensidade da corrente e freqüência terapêutica.

Palavras-chave: estimulação elétrica, marcha, paralisia cerebral, paresia.

\begin{abstract}
One of the most important alterations in gait of patients with sequel of spastic hemiparetic cerebral palsy is the equine foot, which, in general, is caused by muscles weakness. Several non-invasive procedures are used to treat these children, however the neuromuscular electrical stimulation (NMES) has not been frequently reported as a resource used in the therapy of these patients, which would have as objective the muscular reconditioned, the reduction of spasticity and aid motor learning. This work aimed at verifying possible alterations in the ground reactions forces during gait of children with spastic hemiparetic cerebral palsy, immediately after the use of NMES on the tibialis anterior muscle. Six children, average age $7.83 \pm 3.60$ years old, were selected and stimulation frequency at $50 \mathrm{~Hz}$ was applied on the tibialis anterior muscle with evaluation of the standard gait in the pressure platform before and after NMES. Through the results it was observed that there is no gait improvement with only one application of NMES, when compared before and after NMES, however, more investigations are necessary to continue this study with emphasis in motor learning, intensity, and therapeutic frequency.

Key-words: electric stimulation, gait, cerebral palsy, paresis.
\end{abstract}




\section{I ntrodução}

A marcha humana é definida como uma seqüência repetitiva de movimentos dos membros inferiores, que move o corpo para frente, enquanto simultaneamente mantém a estabilidade no apoio. É uma função automática em que há uma adaptação continua do tônus postural, em resposta às constantes mudanças da base de suporte [1]. Para que seja funcional, deve haver uma correlação harmônica entre a ação coordenada das funçôes nervosas, musculares e esqueléticas. Logo, quaisquer condiçóes que perturbem esta correlação, podem resultar em uma marcha dita patológica, trazendo prejuízos primários, ou mecanismos compensatórios secundários à marcha [2].

Uma das alteraçóes mais comuns que influenciam negativamente a marcha normal é o pé eqüino, muito presente em portadores de hemiplegia [3]. A perda da capacidade de recrutar seletivamente o músculo tibial anterior durante a marcha pode levar a uma diminuição do primeiro pico de força reação solo, da taxa de aceitação do peso, tanto no comprimento, como na cadência do passo e conseqüentemente da velocidade da marcha [4].

A conseqüência prática do pé eqüino é uma marcha lenta, insuficiente e instável, o que irá levar o indivíduo a uma inabilidade em participar completamente de sua vida funcional, visto que a mobilidade é parte fundamental da conservação da independência e um atributo importante da qualidade de vida [3].

As condutas fisioterapêuticas, por meio da cinesioterapia, alongamentos, fortalecimento muscular e posicionamento postural adequado visam tornar os músculos menos responsivos a espasticidade, objetivando assim a melhora da funçáo do membro comprometido [5].

Evangelista et al. [6] afirmam que a estimulação elétrica neuromuscular (EENM) tem sido, ao lado da cinesioterapia, um dos recursos mais amplamente utilizados na prática clínica do fisioterapeuta. Guirro et al. [7] complementam dizendo que a EENM pode ser empregada para acelerar o processo de recuperação, mas não deve ser considerada um substituto aos tratamentos convencionais, e sim, um complemento à terapia.

A EENM é uma forma de eletroterapia capaz de produzir contraçóes musculares em músculos privados de controle central, com objetivos funcionais. Este nunca será recurso terapêutico único no tratamento de uma criança com paralisia cerebral, sempre deve fazer parte de um conjunto de procedimentos orientados individualmente, pois uma fisioterapia bem conduzida, deve propor exercícios destinados a estimular a movimentação voluntária e reduzir a espasticidade, devendo utilizar vários tipos de estimulaçáo sensitiva e sensorial $[8,9]$.

O objetivo da EENM em programa de reabilitação do paciente portador de paralisia cerebral do tipo hemiparesia espástica enfatiza o recondicionamento muscular, a redução de espasticidade e o auxílio na aprendizagem motora, devido à incapacidade de realizar movimentos de dorsiflexão do pé, sendo fator agravante no padrão postural anormal durante a marcha. $\mathrm{Na}$ forma hemiparética, os músculos dorsiflexores são os mais freqüentemente estimulados para a promoção da marcha. Contudo, a maioria dos trabalhos que comprova sua eficácia, está relacionada a pacientes adultos, sendo poucos os estudos que comprovam a efetividade do método quando utilizados em crianças portadoras de paralisia cerebral do tipo hemiparesia espástica.

Por isso, este trabalho se justifica no intuito de verificar as possíveis alteraçôes no comportamento das forças reação solo durante a marcha de crianças portadoras de paralisia cerebral do tipo hemiparética espástica, imediatamente após o uso de EENM aplicadas sobre o músculo tibial anterior.

\section{Materiais e métodos}

Participaram deste estudo seis pacientes portadores de Paralisia Cerebral do tipo Hemiparesia Espástica, com idade entre quatro e doze anos $(7,83 \pm 3,60)$, que já realizam acompanhamento fisioterapêutico, sendo destes dois do sexo feminino e quatro do sexo masculino.

O experimento foi realizado após aprovação do Comitê de Ética em Pesquisa (COEP) do Centro Universitário Nove de Julho - UNINOVE (segundo a resolução CNS 196/96, do Conselho Nacional de Saúde, de 10/10/96). Todos os voluntários foram informados do protocolo de estudo e dada orientação sobre o procedimento a que seriam submetidos, além de assinarem o Termo de Consentimento livre e esclarecido.

Adotou-se como critério de inclusão crianças portadoras de paralisia cerebral do tipo hemiparesia espástica, que apresentavam marcha independente, sem auxilio de órteses, dentro do nível 1 [10] da escala GM (General Moviment), entre os graus 1+ e 2 da escala de Ashworth modificada [11], com cognitivo preservado e que suportavam sensitivamente a estimulação elétrica.

Para a realização da eletroestimulação os eletrodos foram posicionados sobre o ponto motor do músculo tibial anterior no membro inferior hemiparético, sendo utilizada uma corrente quadrada e bifásica, com freqüência pré determinada em $50 \mathrm{~Hz}$, largura de pulso de $100 \mu$ seg, sustentação e repouso da contração de $5 \mathrm{seg}$, por 20 minutos.

As crianças foram orientadas a realizar a contração muscular juntamente com a estimulação elétrica para favorecer o movimento e o aprendizado motor, objetivo este buscado com a referida modalidade terapêutica.

O protocolo de avaliaçáo proposto constou do sistema MatScan, que consiste em uma plataforma de pressáo, onde foram coletados dados sobre as características de marcha adotada pelas crianças, em seguida aplicada eletroestimulação por 20 minutos no músculo tibial anterior no membro inferior hemiparético, e realizada, imediatamente após, uma nova avaliaçấo do padrão de marcha na plataforma de pressão. 
Os dados da força de reação ao solo foram calculados pelo sistema MatScan, o qual possui transdutores piezoelétricos sensíveis à pressão exercida na direção vertical. Utilizamos um conversor de 12 bits de resolução e o sinal de força de reaçáo ao solo foi amostrado em uma freqüência de $60 \mathrm{~Hz}$, e filtrado com filtro Butterworth de $4^{\circ}$ ordem digital com freqüência de corte de $5 \mathrm{~Hz}$.

Para a análise dos dados foram selecionados, para cada série temporal de força de reaçáo ao solo: a) os valores do primeiro pico de força vertical máxima (contado do calcanhar no solo - Fy1); b) segundo pico de força vertical máxima (retirada do pé- Fy2); c) força vertical mínima (Fymin); ed) valor de inclinaçáo da curva de força, até o seu pico superior, denominado taxa de aceitaçáo do peso (valor máximo da força vertical passiva dividido pelo tempo decorrido até esse valor ser alcançado).

A plataforma não permite apenas a análise das forças verticais de reação do solo, como também a extração da velocidade da marcha, cadência, e comprimento da passada.

Os dados relacionados a FY1, Fy2 e Fymin foram normalizados pelo peso corporal do voluntário, e expressos pela unidade $\mathrm{N} / \mathrm{kg}$.

Para a análise das variáveis de força reação solo foram calculados, em princípio, os valores médios destes parâmetros de cada uma das coletas realizadas para cada indivíduo, contendo cada uma das coletas uma série de valores correspondentes a cada passada adquirida durante a marcha.

Destes valores médios correspondentes às coletas, foi calculada uma única média representando o valor médio de cada parâmetro para cada grupo analisado.

Para análise estatística inferencial, foi utilizado o teste estatístico não paramétrico de Wilcoxon para dados pareados [12].

\section{Resultados}

A Tabela I mostra os dados demográficos dos participantes. Os voluntários eram crianças, com idade entre 4 e 12 anos, sendo quatro do sexo masculino e dois do sexo feminino, média de idade semelhante entre os gêneros, porém com massa e altura de $16,6 \mathrm{~kg}$ e $1,20 \mathrm{~cm}$ para o gênero masculino e $9 \mathrm{~kg}$ e $1,05 \mathrm{~cm}$ para o gênero feminino.

Tabela I - Dados demográficos dos participantes $(n=06)$.

\begin{tabular}{lllll}
\hline Sujeitos & Gênero & $\begin{array}{l}\text { Idade } \\
\text { (anos) }\end{array}$ & Massa $(\mathrm{kg})$ & Altura $(\mathrm{m})$ \\
\hline 1 & $\mathrm{M}$ & 4 & 17,2 & 1,2 \\
2 & $\mathrm{M}$ & 7 & 13,2 & 1,2 \\
3 & $\mathrm{M}$ & 9 & 15 & 1,25 \\
4 & $\mathrm{M}$ & 10 & 21 & 1,2 \\
Média & & 7,5 & 16,6 & 1,20 \\
5 & $\mathrm{~F}$ & 5 & 7 & 1,0 \\
6 & $\mathrm{~F}$ & 12 & 11 & 1,11 \\
Média & & 8,5 & 9 & 1,05 \\
\hline
\end{tabular}

A Tabela II representa os valores médios e desvios padrão obtidos da força reação do solo referentes à força vertical do membro comprometido durante o ciclo completo da passada, pré e pós EENM com a freqüência de estimulação pré-determinada.

Tabela II - Valores médios com respectivos desvios padrão e valor de p, 5\% de probabilidade das 10 passadas de cada um dos seis voluntários, pré e pós o uso de estimulação elétrica neuromuscular (EENM).

\begin{tabular}{llll}
\hline Variáveis & Pré EENM & $\begin{array}{l}\text { Imediata- } \\
\text { mente Pós } \\
\text { EENM }\end{array}$ & $\begin{array}{l}\text { Valor } \\
-\mathbf{p}\end{array}$ \\
\hline $\begin{array}{l}\text { Pico da força vertical } \\
\text { máxima (Fy1 x PC*) }\end{array}$ & $0,86 \pm 0,01$ & $0,81 \pm 0,03$ & $0,05^{* *}$ \\
$\begin{array}{l}\text { Pico da força vertical } \\
\text { máxima (Fy2 x PC*) }\end{array}$ & $0,82 \pm 0,02$ & $0,82 \pm 0,02$ & $0,06^{* *}$ \\
$\begin{array}{l}\text { Pico da força vertical } \\
\text { mínima (Fymin x }\end{array}$ & $0,75 \pm 0,01$ & $0,76 \pm 0,05$ & $0,05^{* *}$ \\
$\begin{array}{l}\text { PC*) } \\
\begin{array}{l}\text { Taxa de aceitação } \\
\text { do peso }\end{array}\end{array}$ & $7,38 \pm 0,39$ & $7,85 \pm 0,25$ & $0,65^{* *}$ \\
*onde PC significa Peso Corporal & & \\
** não significante & & &
\end{tabular}

Analisando os valores das variáveis da força reação solo, durante o ciclo da marcha, não podemos observar alteraçóes destes pré e imediatamente pós EENM, $(\mathrm{p}<0,05)$.

A velocidade da marcha, cadência e comprimento da passada também náo apresentaram nenhuma diferença estatisticamente significante $(\mathrm{p}=0,53)$.

\section{Discussão}

Diversos fatores devem ser considerados na análise das evidências disponíveis na literatura sobre a eficácia das abordagens terapêuticas não-medicamentosas e não-cirúrgicas na promoção da marcha em crianças portadoras de PC [13]. A EENM é um importante complemento para inúmeros programas de tratamento utilizados pela fisioterapia. Este instrumento pode ser empregado para acelerar processos de recuperaçáo em várias áreas, não devendo, entretanto, ser considerado um substituto para os tratamentos tradicionais [7].

Uma das respostas fisiológicas encontradas diante do uso da EENM é o aumento e melhora do recrutamento de fibras musculares, sendo que a maioria delas, senáo todas, apresenta uma resposta a eletroestimulação diferenciada do movimento ativo normal, que só pode recuperar uma porcentagem das fibras [14] proporcionando uma melhora na performance do indivíduo [6].

Segundo Evangelista et al. [6], com o uso da EENM consegue-se mais facilmente as mudanças nas características das fibras musculares. Esta modificação é dependente principalmente da freqüência que se despolariza o nervo motor por meio 
da corrente elétrica, sendo 50 a $150 \mathrm{~Hz}$ ideal para trabalhar músculos dinâmicos (fásicos) e/ou garantir que as fibras tornemse brancas, e que 20 a $30 \mathrm{~Hz}$ é ideal para trabalhar músculos estáticos (tônicos) e/ou garantir que as fibras brancas tornem-se vermelhas. Kramer [14] afirma que existe uma relaçâo direta entre a ativação de unidades motoras e a tensão gerada pelo músculo, sendo estas freqüências em contraçôes voluntárias máximas contínuas encontradas em torno de 30 a $70 \mathrm{~Hz}$.

Em suas pesquisas, Guirro et al. [7] concluem que tanto correntes de média freqüência como as de baixa freqüência apresentam resultados similares e satisfatórios quanto ao ganho de força em indivíduos sadios.

Diversos estudos sustentam a tese de que parte do efeito do treinamento está no aprendizado motor ou na facilitação neural em função de um número maior de unidades motoras ativadas, aumento na taxa e impulsos ou um padráo mais eficiente de recrutamento [7].

A estimulação elétrica neuromuscular (EENM) tem se mostrado um recurso muito eficaz no auxilio do tratamento do pé eqüino, mediante a hemiparesia e a espasticidade. Porém, a maioria dos trabalhos que comprova sua eficácia está relacionada a pacientes adultos, sendo poucos os estudos que comprovam a efetividade do método quando utilizados em crianças portadoras de paralisia cerebral do tipo hemiparesia espástica.

O estudo de Neilson e Caughey [15] mostra que com o treinamento houve evoluçáo da marcha com biofeedback de assistência dorsiflexora, em que os pacientes puderam regular a espasticidade e ocasionalmente o espasmo muscular sendo a motivaçáo dos pacientes um fator importante.

As crianças por nós avaliadas foram orientadas a realizar o movimento de dorsiflexão do tornozelo durante a aplicação da EENM com objetivo de aprendizado motor do movimento, mas observamos que apresentavam grande distração durante a aplicação, não realizando muitas vezes o movimento solicitado obtendo insucesso no aprendizado, segundo os dados obtidos em nosso estudo.

Para Camargo et al. [16], a efetividade da técnica de EENM está relacionada com a intensidade de corrente suportada pelo indivíduo. Brasileiro e Villar [17] e Guirro et al. [7] complementam dizendo que quanto maior a intensidade de corrente suportada pelo indivíduo, maior será o incremento de força muscular, e em conseqüência, aumento do recrutamento das unidades motoras.

Acreditamos que a intensidade de corrente por nos utilizada não tenha sido a máxima suportada pelas crianças, pois estas apresentaram pouca tolerância à estimulaçáo elétrica. Observou-se que à medida que aumentávamos a intensidade, as crianças apresentavam-se agitadas e inquietas, referindo incomodo durante a passagem da corrente. Durante a aplicação da estimulaçáo elétrica foram respeitados os limites de cada criança quanto à intensidade da corrente.

Diferente de nosso estudo, Martinez et al. [18] obtiveram melhora da funcionalidade da extremidade hemiparética em pacientes com PC após uso de doses baixas de toxina botulínica e EENM de baixa freqüência por 12 sessōes, utilizadas em um mesmo programa de reabilitação.

Já o estudo de Pease [19] foi realizado com o medicamento baclofen, por seis semanas, associado a um programa de terapia física, e, após, realizou-se terapia com EENM duas a três vezes por semana, durante três meses, associada a exercícios de fortalecimento intercalados nos outros dias, gerando uma marcha eficiente com um significante benefício na melhora da velocidade desta.

Carmick [20] também encontrou resultados satisfatórios quanto a utilização da estimulação elétrica no músculo tríceps sural, combinada ao tratamento fisioterapêutico de pacientes portadores de diplegia espástica. Seus resultados apontaram melhora da marcha, equilíbrio, postura e amplitude de movimento ativo e passivo de tornozelo, náo observando aumento da espasticidade. Seu estudo se baseou em duas crianças, que apresentavam pé eqüino e alterações significativas de marcha. Trimble e Enoka [21] sugerem que a melhora do equilíbrio se deve a um aumento da propriocepçáo gerada pela estimulação elétrica somada ao treino de marcha.

Staub et al. [22] apontam como achado principal de sua pesquisa a melhora da funcionalidade e da marcha, gerada pela estimulação elétrica, baseado em quatro crianças portadoras de paralisia cerebral do tipo diparesia espástica, que realizavam o protocolo de estimulação elétrica três vezes por semana nos músculos tibial anterior e reto femoral associados ao treino de marcha após cada sessão.

Segundo o estudo de Gibertoni et al. [23], o uso da eletroestimulação aplicada nos dorsiflexores de tornozelo, de pacientes portadores de hemiparesia gerou melhora e promoção da marcha funcional.

Porém, Sommerfelt et al. [24] realizaram um estudo randomizado, concluindo que a estimulação elétrica não ofereceu efeitos significativos na função motora e melhora da marcha de pacientes diplégicos. Os autores utilizaram a estimulação elétrica transcutânea em nível de sub-contração.

Em 2004, Brasileiro e Salvini [25] também não observaram resultados que indicassem ganho de força nos indivíduos por eles estudados. Para a realização deste trabalho, os autores compararam músculos com grau de força normal e músculos com déficit de força, sendo que ambos não apresentaram melhora ou aumento da força muscular após a realização da estimulação elétrica. Não foram encontrados, em seu trabalho, dados referentes aos parâmetros por eles utilizados.

Brasileiro e Salvini [25] afirmam que os estudos que envolvem o uso da EENM são bastante controversos, pois além dos diferentes procedimentos metodológicos, os parâmetros manipuláveis clinicamente nos geradores de corrente não obedecem a um padrão uniforme, o que torna difícil a comparação dos resultados obtidos em diferentes estudos, talvez explicando as grandes divergências nas conclusões obtidas em diferentes experimentos. 
Nossos resultados não foram estatisticamente significativos de acordo com os dados obtidos, mostrando que uma única aplicação de EENM não demonstra melhora do padrão de marcha. Alguns fatores são sugeridos para o insucesso quanto a dados que pudessem validar o uso da EENM na marcha de pacientes com seqüela de $\mathrm{PC}$ do tipo hemiparesia espástica: falta de aprendizado motor devido a grande distraçáo durante a aplicação; a intensidade da corrente, que talvez não tivesse sido a máxima suportada pelas crianças, não resultando em uma contração tão eficaz; e a freqüência terapêutica, ou seja, falta da continuidade do tratamento, uma vez que utilizamos apenas uma única sessão como protocolo neste estudo.

\section{Conclusão}

De acordo com os dados obtidos nesse trabalho, podemos afirmar que o recondicionamento muscular, a redução de espasticidade e o auxílio na aprendizagem motora com uma única aplicação, sem continuidade de tratamento, não difere quando comparado pré e imediatamente pós EENM.

Observou-se que o padrão da força reação solo, obtido durante a marcha, não se mostrou estatisticamente significante. Entretanto, faz-se necessário a continuação desse estudo com ênfase em aprendizado motor, intensidade da corrente e freqüência terapêutica.

\section{Agradecimentos}

Agradecimento ao apoio FAPESP pela Bolsa de Iniciação Científica, processo no 2005/00502-0.

\section{Referências}

1. Perry J. Gait Analysis: normal and pathological function. Thorofare: Slack; 1992.

2. Corrêa FI, Soares F, Andrade DV, Gondo RM, Peres JA, Fernandes $\mathrm{AO}$. Muscle activity during gait following stroke. Arq Neuropsiquiatr 2005;63(3b):847-51.

3. Burridge JV, McLellan DL. Relation between abnormal patterns of muscle activation and response to common peronel nerve stimulation in hemiplegia. J Neurol Neurosurg Psychiatry 2000;69:353-61.

4. Chen YL, Kuo TS, Lai JS. The development of a closed-loop controlled functional electrical stimulation (FES) in gait training. J Med Technol 2001;25:41-48.

5. Resende CMG, Nascimento VF, Leite JMRS. Eficácia da toxina botulínica tipo-A associada à fisioterapia em uma criança hemiplégica espástica. Neurociências 2005;13:35-39.

6. Evangelista AR, Gravina GA, Borges FS, Vilardi NP. Adaptação da característica fisiológica da fibra muscular por meio da eletroestimulação. Fisioter Bras 2003;4:326-34.

7. Guirro R, Nunes CV, Davini R. Comparação dos efeitos de dois protocolos de estimulação elétrica neuromuscular sobre a força muscular isométrica do quadríceps, 2000. Rev Fisioter Univ São Paulo 2000;7:15-19.
8. Lianza S. Medicina da reabilitação. Rio de Janeiro: Guanabara Koogan; 1995.

9. Diament A, Cypel S. Neurologia Infantil. São Paulo: Atheneu; 1996.

10. Gowland C, Boyce WF, Wright V, Russel DJ, Goldsmith CH, Rosenbaum PL. Reliability of the gross motor performance measure. Phys Ther 1995;75:597-602.

11. Rodriguez IM, Torres GC, Rodriguez G. Eficacia de la aplicación del programa de rehabilitación física aplicado en la clínica de neuropediatría para la disminución de la espasticidad y el aumento de la capacidad motora en niños con parálisis cerebral. Lecturas: EF y Deportes [online] 2003;67:30-35.

12. Pagano M, Gauvreau K. Princípios de bioestatística. 2a ed. São Paulo: Thomson; 2004. 506 p.

13. Chagas PSC, Mancini MC, Barbosa AP, Silva PTG. Análise das intervençôes utilizadas para promoção da marcha em crianças portadoras de paralisia cerebral: uma revisão sistemática da literatura. Rev Bras Fisioter 2004;8:155-63.

14. Kramer J. Effect of electrical stimulation current frequencies on isometric knee extension torque. Phys Ther 1987;67:31-38.

15. Neilson PD, McCaughey J. Self-regulation of spasm and spasticity in cerebral palsy, 1982. J Neurol Neurosurg Psychiatry 1982;45:320-30.

16. Camargo LC, Minamoto VB, Noronha MA, Castro CES, Salvani TF. A estimulação elétrica neuromuscular do tibial anterior náo altera a morfologia dos músculos sólios (antagonistas) e extensor digital longo (sinergista) do rato. Rev Fisioter Univ São Paulo 1998;3:120-26.

17. Brasileiro JS, Villar AFS. Comparação dos torques gerados pela eletroestimulação e contração muscular voluntária no músculo quadríceps femoral. Rev Bras Fisioter 2000; 3:75-81.

18. Martinez LD, Saldaña MEA, Cabrera MGV, Duarte AM, Reyes GR, Aldo AM. Utilidad de la estimulación eléctrica neuromuscular posterior a la aplicación de toxina botulínica en niños con hemiparesia espástica. Rev Mex Med Fis Rehabil 2005;17:16-22.

19. Pease WSMD. Therapeutic electrical stimulation for spasticity: quantitative gait analysis. Am J Phys Med Rehabil 1998;77:35155.

20. Carmick J. Managing equinus in children with cerebral palsy: electrical stimulation to strengthen the triceps sural muscle. Dev Med Child Neurol 1995;37:965-75.

21. Trimble MH, Enoka RM. Mechanisms underlying the training effects associated with neuromuscular electrical stimulation. Phys Ther 1991;71:273-9.

22. Staub ALP, Rotta NT, Mahmud MAI, Svirski AS, Santos AC, Fonteles VR et al. Efeitos da EENM em pacientes com paralisia cerebral do tipo diplegia espástica. Fisioter Bras 2005;6:2531.

23. Gibertoni F, Lopes J, Scoton MK. Análise da marcha hemiplégica após eletroestimulaçáo funcional. Reabilitar 2003;5:18:22.

24. Sommerfel TK, Markestad T, Berg K, Saetisdal I. Therapeutic electrical stimulation in cerebral palsy: a randomized, controlled, crossover trial. Dev Med Child Neurol 2001; 43:609-13.

25. Brasileiro JS, Salvini TF. Limites da estimulação elétrica neuromuscular no fortalecimento de músculos esqueléticos saudáveis e com déficit de força. Fisioter Bras 2004;5:224-230. 